\title{
Widefield fluorescence microscopy with extended resolution
}

\author{
Andreas Stemmer $\cdot$ Markus Beck $\cdot$ Reto Fiolka
}

Accepted: 31 August 2008/Published online: 23 September 2008

(C) Springer-Verlag 2008

\begin{abstract}
Widefield fluorescence microscopy is seeing dramatic improvements in resolution, reaching today $100 \mathrm{~nm}$ in all three dimensions. This gain in resolution is achieved by dispensing with uniform Köhler illumination. Instead, non-uniform excitation light patterns with sinusoidal intensity variations in one, two, or three dimensions are applied combined with powerful image reconstruction techniques. Taking advantage of non-linear fluorophore response to the excitation field, the resolution can be further improved down to several $10 \mathrm{~nm}$. In this review article, we describe the image formation in the microscope and computational reconstruction of the high-resolution dataset when exciting the specimen with a harmonic light pattern conveniently generated by interfering laser beams forming standing waves. We will also discuss extensions to total internal reflection microscopy, non-linear microscopy, and three-dimensional imaging.
\end{abstract}

Keywords Structured illumination - Extended resolution - Widefield fluorescence microscopy

\section{Introduction}

An ever-growing selection of highly specific fluorescent markers binding to or being expressed as part of single molecules and molecular assemblies make light microscopy the method of choice to study molecular architecture and dynamics in cells (Taylor and Wang 1980; Stephens

\footnotetext{
A. Stemmer $(\bowtie) \cdot$ M. Beck · R. Fiolka

Nanotechnology Group, Department of Mechanical and Process

Engineering, ETH Zurich, Tannenstrasse 3,

8092 Zurich, Switzerland

e-mail: astemmer@ethz.ch
}

and Allan 2003). Particularly attractive is the possibility to simultaneously observe a multitude of cellular constituents that are labeled with markers of distinct emission wavelengths to quantify their spatial and temporal distribution and possible colocalization. Although the numerical aperture (NA) of the objective and the wavelength of light physically limit the attainable resolution, a fact described by Ernst Abbe already in 1870 (Abbe 1873), separations much smaller than this limit can be determined between two isolated markers emitting on two different wavelengths because the position of each signal source can be measured independently with very high precision. For each wavelength, however, the width and axial extent of the point spread function (PSF), i.e., the image of an arbitrarily small light emitter, determines the observable size of an object and how close two objects can still be resolved. In practice, the point resolution of a standard widefield light microscope with well corrected high-NA objectives amounts to approx $230 \mathrm{~nm}$ laterally and $800 \mathrm{~nm}$ in axial direction, which is not sufficient to resolve a large class of biological structures. Hence, much effort has been devoted to extend the optical resolution beyond the classical diffraction limit.

This review article focuses on techniques known as structured illumination that extends the resolution of widefield fluorescence microscopes to $100 \mathrm{~nm}$ and below. Unlike Köhler illumination that aims at uniformly illuminating the field of view, in structured illumination the specimen is illuminated with a light pattern exhibiting bright and dark portions. Patterns with sinusoidal (harmonic) intensity variations in one, two, or three dimensions are of particular importance since they are readily created by interfering laser beams. Furthermore, harmonic excitation patterns lend to powerful image reconstruction techniques in Fourier space. Figure 1 illustrates, in comparison with confocal and atomic force microscopy, the 

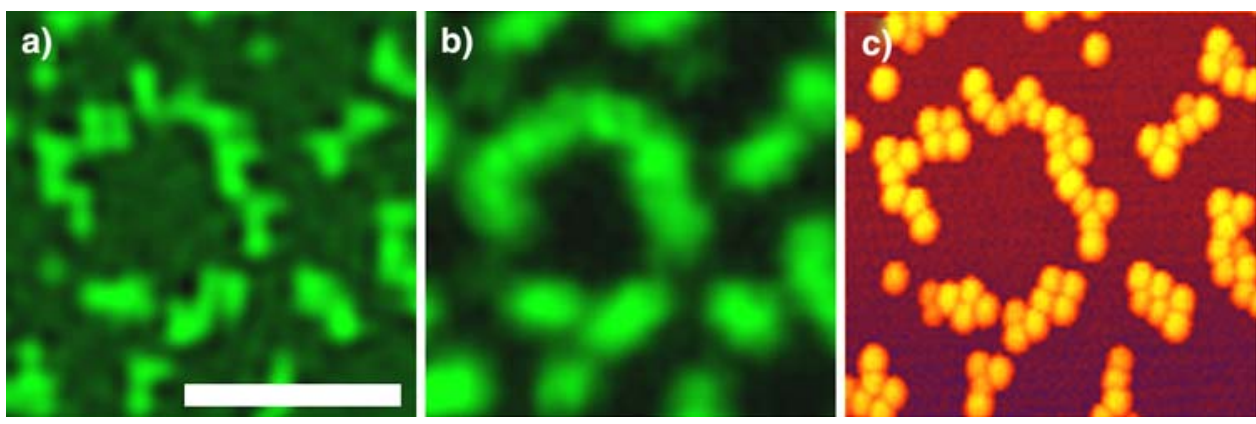

Fig. 1 Identical area of a sample of 100-nm diameter fluorescent polystyrene beads imaged with different techniques (Bar $1 \mu \mathrm{m}$.) a Individual beads are resolved by the HELM technique implemented on a Zeiss Axiovert microscope with a Plan Apo $\times 63$ 1.4 NA objective. b The confocal image was recorded on a Leica NTSP with

gain in resolution achievable when illuminating the specimen with a two-dimensional harmonic light pattern. We will refer to this technique as harmonic excitation light microscopy (HELM).

In the following sections, we will discuss the concepts and developments that led to the dramatic resolution improvements feasible in widefield fluorescence microscopy today. We will outline image formation in the microscope and computational reconstruction of the high-resolution dataset when exciting a fluorescent specimen with a harmonic light pattern. The corresponding illumination set-ups are also explained. Finally, we provide an overview of further developments exploiting non-linearities in the response of fluorophores to reach a resolution of a few $10 \mathrm{~nm}$ with visible light and standard microscope objectives.

\section{Developments toward extended resolution}

The confocal microscope invented by Marvin Minsky in 1955 marks the first practical concept to extend the classical resolution limit (Minsky 1988). Using a pinhole to reject out-of-focus light, the optical sectioning capability and hence axial resolution was dramatically improved over standard widefield microscopes (White et al. 1987). Theoretically, a small pinhole also increases lateral resolution up to a factor of 1.4. In practice, however, this resolution enhancement is seldom realized since resolution is traded off against signal strength when the pinhole is closed down.

In 1963, Lukosz and Marchand introduced a general concept to increase the optical resolution using structured illumination instead of uniform light as effected by Köhler illumination (Lukosz 1966). Structured illumination, e.g., by a grid pattern, improves spatial resolution at the cost of temporal resolution since several images with shifted illumination pattern have to be acquired in order to extract more information. In this respect, the confocal microscope a Leica Plan Apo $\times 1001.4$ NA objective. c Reference image recorded with an atomic force microscope (TopoMetrix Accurex II MS). Reprinted with permission from (Frohn et al. 2000). Copyright (2000) National Academy of Sciences, USA

represents a limiting case of structured illumination, since a spot of light is scanned across the field of view and the signal is acquired for each position.

Only in 1993, Lanni and Bailey introduced a first application of structured illumination, namely standing wave fluorescence microscopy (Bailey et al. 1993). In their set-up, two interfering laser beams generated a standing wave in axial direction. The alternating nodal (dark) and antinodal (bright) planes parallel to the object plane enabled selective excitation of individual sections in the sample. However, this technique was only suitable for very thin samples in the range of the period of the standing wave. In thicker samples, nodal planes above and/or below the focal plane create out-of-focus blur due to the poor axial resolution of widefield microscopes.

Gustafsson et al. (1995) improved the basic concept of standing wave microscopy by using two objectives for illumination and detection. In his set-up, the focal plane is selectively excited by the interference of counter-propagating light beams originating from an incoherent source. Additionally the images collected by the two objectives are coherently superimposed on a CCD detector. I5M microscopy achieved a sevenfold higher axial resolution than conventional microscopes. In parallel, Hell and co-workers introduced 4Pi microscopy, the point scanning analog to I5M that also employs two objectives (Hell and Stelzer 1992). Both the techniques demand a very careful alignment of the objectives and the optical train used for image formation, and the image is reconstructed by computational post processing. For ease of use, the 4Pi microscope has to be operated in the two-photon excitation regime (Hell and Stelzer 1992).

Surprisingly, these concepts only aimed at increasing the axial resolution, which of course is an important issue when studying three-dimensional objects, but the lateral resolution remained within the classical limit.

Lateral resolution, as suggested by Lukosz, can be improved in a similar way by illuminating the object with 
standing waves extending in lateral direction. A first practical implementation of this concept was published by Heintzmann and Cremer (1999), and later Gustafsson (2000) and Frohn et al. (2000) independently demonstrated microscope set-ups reaching twice the optical resolution. So and co-workers recognized the possibility to further extend the lateral resolution by applying standing wave illumination to total internal reflection fluorescence (TIRF) microscopy (Cragg and So 2000; Chung et al. 2006, 2007).

In 1994, Hell and co-workers took advantage of optical non-linearities to fundamentally improve resolution in a point scanning microscope (Hell and Wichmann 1994; Klar et al. 2000). To this end, they depleted the fluorescence at the rim of a focused spot by stimulated emission. Later, Heintzmann et al. (2002) extended the concept of resolution enhancement by non-linear phenomena to widefield microscopy and structured illumination, and Gustafsson (2005) succeeded in experimentally demonstrating a lateral resolution of $50 \mathrm{~nm}$. Techniques relying on non-linear fluorophore response typically require long acquisition times and may suffer from increased photobleaching and phototoxicity, limiting their application to biological specimens unless particularly stable fluorophores are employed.

Despite earlier suggestions (Heintzmann and Cremer 1999; Gustafsson et al. 2000; Frohn et al. 2001), due to technical challenges simultaneous enhancement of lateral and axial resolution yielding a near-isotropic resolution of about $100 \mathrm{~nm}$ using structured light was only recently achieved by Gustafsson and co-workers (Gustafsson et al. 2008; Schermelleh et al. 2008; Shao et al. 2008). The remarkable gain in resolution becomes very apparent in complex biological specimens.

\section{Image formation}

In fluorescence microscopy, image formation is mathematically described in the framework of a linear shift invariant system. For such a system, the complete signal transfer from object to image is described by the PSF (Fig. 2, see also Fig. 10a). Every object may be considered as being composed of a set of infinitesimally small point emitters, each of which is blurred by the PSF upon imaging, resulting in a loss of resolution. The spatial extent of the PSF is the key factor limiting resolution in light microscopy to $\delta x=\lambda /(2 \mathrm{NA})$, where $\lambda$ denotes the wavelength of light in vacuum.

Alternatively, one may describe the imaging process in Fourier (reciprocal/frequency) space. Any object can be decomposed into a sum of sinusoids of different spatial frequencies and amplitudes. The objective, however, can only collect a limited set of low spatial frequencies. The so-

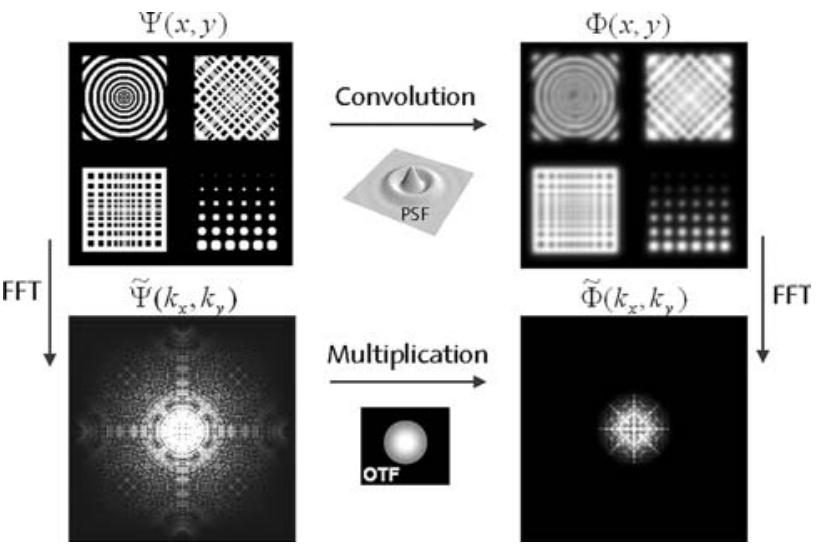

Fig. 2 Image formation in fluorescence microscopy. In real space (top row), image formation is described by a convolution of the point spread function (PSF) with the emitted light from the object $\Psi(x, y)$. In reciprocal space (bottom row), the image spectrum $\tilde{\Phi}\left(k_{x}, k_{y}\right)$ is formed by multiplication of the object spectrum $\tilde{\Psi}\left(k_{x}, k_{y}\right)$ with the object transfer function (OTF)

called optical transfer function (OTF) is defined as the region in Fourier space that has non-zero values (Fig. 2, see also Fig. 10b). The bigger the extent of the OTF, the higher is the resolution. Figure 2 illustrates how the image of an object is blurred by the PSF and, correspondingly, how the object spectrum is filtered by the OTF.

\section{HELM theory}

\section{Real space}

How does a harmonic excitation pattern, e.g., the standing wave created by interfering laser beams, lead to higher resolution? The underlying physics is frequency mixing, i.e., the transformation of the object's spatial frequencies into a set of lower and a set of higher frequencies by subtracting or adding the spatial frequency of the harmonic excitation pattern, respectively. In fluorescence microscopy, we deal with a multiplication of the illumination pattern with the labeled specimen. Hence we may apply Euler's formula to describe the multiplication of the object frequencies $k_{1}$, with the harmonic illumination pattern of spatial frequency $u: \cos \left(k_{1} x\right) \cos (u x)=1 / 2\left[\cos \left(k_{1}+u\right)+\right.$ $\left.\cos \left(k_{1}-u\right)\right]$.

The resulting signal is separated into a component with higher frequencies $\left(k_{1}+u\right)$ and, more interesting for light microscopy, into a component with lower frequencies of $k=\left(k_{1}-u\right)$. Provided $k$ remains within the microscope's passband, i.e., the region with non-zero OTF, the low frequency component can carry higher spatial-frequency information into the passband than predicted by the objective's resolution limit. One should note, however, that one could not properly retrieve this higher-frequency 
information from a single image. Obviously, with a harmonic excitation pattern, we do not uniformly illuminate the specimen and we must acquire additional images with shifted pattern that excites previously dark portions. As we will see below, the additional images are also required to retrieve the high-frequency information. Now we can calculate the highest spatial frequency transmitted through an optical passband with cut-off frequency $k_{\mathrm{c}}=2 \mathrm{NA} / \lambda$ : $k_{\max }=k_{\mathrm{c}}+u$. For $u=k_{\mathrm{c}}$ this leads to a doubling in resolution. The high-resolution information may be retrieved in real space by comparing images acquired with different shifts of the illumination pattern (So et al. 2001).

\section{Fourier space}

To illustrate the effect of harmonic excitation in Fourier domain we schematically depict an image spectrum (Fig. 3a, filled squares) together with the passband of the microscope (shaded circle). The smallest squares, representing high spatial-frequency information, lie outside of the passband and cannot be retrieved in conventional microscopy. Harmonic excitation creates additional copies of the image spectrum (open squares in Fig. 3a), shifted by the vector $u=2 \pi / \Lambda$, where $\Lambda$ is the period of the illumination pattern. This shift brings higher spatial frequencies of the image spectrum (open squares) into the passband so they can be detected, albeit at the wrong position in frequency space. Illumination by a two-dimensional (2D) harmonic light field (Fig. 3b) gives rise to a fluorescence spectrum $\tilde{\Phi}$ consisting of a linear combination of five spectral components:

$$
\begin{aligned}
\tilde{\Phi}(\vec{k})= & 4 \tilde{\Psi}_{1}(\vec{k})+e^{-i \Delta \varphi_{x}} \tilde{\Psi}_{2}\left(\vec{k}-\vec{u}_{x}\right)+e^{+i \Delta \varphi_{x}} \tilde{\Psi}_{3}\left(\vec{k}+\vec{u}_{x}\right) \\
& +e^{-i \Delta \varphi_{y}} \tilde{\Psi}_{4}\left(\vec{k}-\vec{u}_{y}\right)+e^{+i \Delta \varphi_{y}} \tilde{\Psi}_{5}\left(\vec{k}+\vec{u}_{y}\right)
\end{aligned}
$$

To reconstruct the extended object spectrum (Fig. 3b), a sequence of $i=1, \ldots, 5$ raw images with different phases of the illumination pattern $\left(\Delta \varphi_{\mathrm{x}}, \Delta \varphi_{\mathrm{y}}\right)_{\mathrm{i}}$ is acquired. The phase steps are chosen such that the sequence of pattern shifts

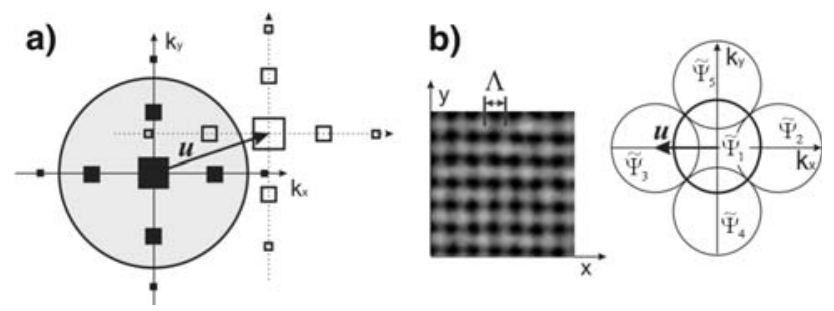

Fig. 3 a Structured illumination adds additional spectral copies (open squares) to the original spectrum (filled squares). The copies are shifted by the vector $\boldsymbol{u}$ out of the center of the passband (shaded circle). This shift brings higher spatial-frequency information (smaller squares) into the passband and contributes to image formation. b Two-dimensional illumination pattern of HELM (left) and reconstructed extended passband (right) will illuminate the entire field of view, e.g., $0^{\circ}$ and $\pm 90^{\circ}$ or $\pm 120^{\circ}$ to reduce bleaching effects in long run experiments. Applying image arithmetic to the image set allows one to separate the spectral components $\tilde{\Psi}_{1, \ldots, 5}$ and correctly rearrange them (Heintzmann and Cremer 1999).

Image reconstruction

To separate and rearrange the spectral components $\tilde{\Psi}_{1, \ldots, 5}$ one must determine the exact period and orientation of the excitation pattern. To this end, one may record a calibration image without fluorescence filters and analyze the pattern by Fourier transformation. The peak positions are obtained by interpolation. A widely used three-point Gaussian fit typically determines the pattern period within $\sim 2 \mathrm{~nm}$, which is not insufficient for reconstruction. Better results are achieved by using the fit value as initial guess to iteratively maximize the mean square error between the recorded image and an analytical cosine pattern. The iteration is stopped when the pattern period reaches a convergence band of $<0.1 \mathrm{~nm}$. Alternatively, one may determine the wave vector $\boldsymbol{u}$ of the illumination pattern from the overlap region of the separated spectral components, i.e., without calibration image (Heintzmann and Cremer 1999; Gustafsson et al. 2000; Gustafsson 2000).

The image spectra of the raw images are compensated by the measured OTF of the microscope. To avoid noise amplification we employ a Wiener filter for deconvolution. The sequence of $i=1, \ldots, 5$ compensated image spectra with known phase shift of the illumination pattern $\left(\Delta \varphi_{\mathrm{x}}\right.$, $\left.\Delta \varphi_{\mathrm{y}}\right)_{\mathrm{i}}$ form an equation system according to Eq. 1. To determine the spectral components $\tilde{\Psi}_{1, \ldots, 5}$ the equation system is solved for each pixel by inverse matrix multiplication. The spectra are then shifted back to the origin of frequency space by applying the Fourier shift theorem in real space, i.e., by multiplying the Fourier back-transform by the corresponding non-stationary phases $\left(e^{ \pm i \vec{u}_{x} \vec{x}}, e^{ \pm i \vec{u}_{y} \vec{x}}\right)$.

The detection of the initial phase of the illumination pattern is a critical step in the reconstruction. In the presence of phase offsets, assuming the initial phase of the pattern to be zero results in wrong weighting of the spectral components. False intensities and a lateral shift of the reconstructed image would result (Schaefer et al. 2004).

The initial phase can be determined from the overlap regions of the different spectral components. To this end, one applies a cross correlation analysis of the phase angles $\theta_{i}=\arctan \left(\operatorname{Im}\left(\tilde{\Psi}_{i}\right) / \operatorname{Re}\left(\tilde{\Psi}_{i}\right)\right)$ between the unshifted spectral component and the shifted components in a finite pixel field of the overlap region, and iteratively maximizes the correlation coefficient (Beck et al. 2008).

Once all spectral components are superimposed into a single data set to form the extended HELM passband, the sharp transition to zero at the rim of the HELM passband 
needs to be smoothed by an apodization function to avoid ringing artifacts in the extended resolution image calculated by Fourier back-transform. Since apodization not only reduces ringing artifacts but also broadens spectral features, we apply a function with constant weighting over most of the HELM passband and a Gaussian decay at the edge to damp the spectrum down to $0.5 \%$ at the limit of the extended OTF (Beck et al. 2008).

The final extended resolution HELM image is obtained after Fourier transforming the extended spectrum into real space. To accommodate the higher resolution, spectra are re-sampled by zero padding. The pixel-size reduction is usually chosen to meet the Shannon-Nyquist criterion. Larger reductions may be used to produce smoother reconstructions.

\section{Instrumentation}

Non-uniform illumination with a harmonic (sinusoidal) excitation pattern is straightforward to configure on the microscope (Fig. 4). A direct way to create a harmonic excitation pattern is to interfere two counter-propagating mutually coherent laser beams. Figure $4 \mathrm{a}$ displays the resulting axial standing wave pattern when the two laser beams are launched through two objectives facing each other (Bailey et al. 1993). The two mutually coherent laser beams are conveniently created by a beam splitter mounted after the laser source. If one allows the laser beams to exit the objectives at an angle relative to the optical axis, standing wave patterns with different orientation and period may be created which is useful when extending harmonic excitation toward 3D microscopy (Heintzmann and Cremer 1999; Gustafsson et al. 2000; Frohn et al. 2001).

To extend lateral resolution, the standing wave pattern of Fig. $4 \mathrm{a}$ needs to be turned by $90^{\circ}$. Figure $4 \mathrm{~b}$ displays a cross-section of a prism launch set-up coupling two pairs (only one pair shown) of interfering laser beams into the specimen chamber to create a two-dimensional grid-like harmonic excitation pattern (Frohn et al. 2000). The laser beams enter the specimen chamber at an oblique angle relative to the cover slide and propagate into the objective to permit observation of the standing wave pattern. This feature facilitates calibration of the illumination pattern (see above) but is not an absolute must.

When illuminating the specimen with two standing wave patterns simultaneously, orientated along the $x$ - and $y$-axis, image analysis is facilitated when the two patterns do not cross-interfere. To this end, one may select s-polarization for the laser beams, which additionally increases the pattern contrast (modulation depth). As a result of the limited number of polarization directions, however, fluorophores with fixed dipol axis may not get excited if orientated in an unfavorable direction.

A harmonic excitation pattern may also be created by projecting a diffractive phase grating into the specimen plane. Figure $4 \mathrm{c}$ illustrates the principle on the example of a two-dimensional phase grating. Selecting only the first diffraction orders and blocking the zero order results in a two-dimensional sinusoidal intensity variation. Blocking the zero order also doubles the spatial frequency of the pattern. The grating approach benefits from a very stable illumination pattern since spatial drifts of the grating are
Fig. 4 Configurations for harmonic excitation. a Standing wave excitation in axial direction using two objectives on opposite sides of the specimen. b Prism-launch for standing wave excitation in lateral direction. The prisms are oil-contacted to the slide. c Grating projection. The first diffraction orders of a phase grating are projected into the specimen plane. d Set-up for HELM in epi-illumination a)

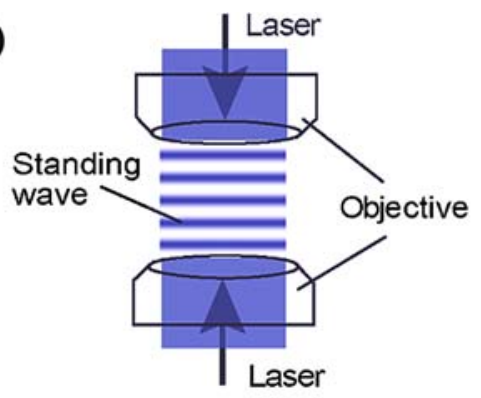

c)

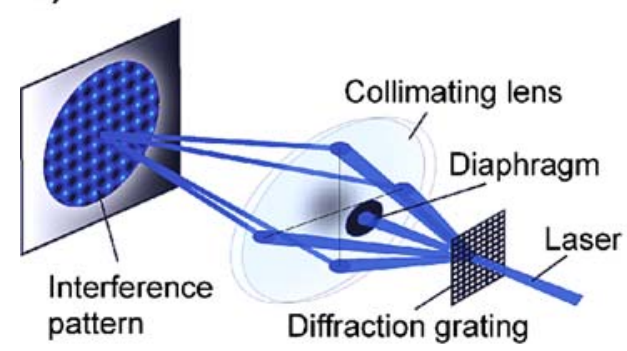

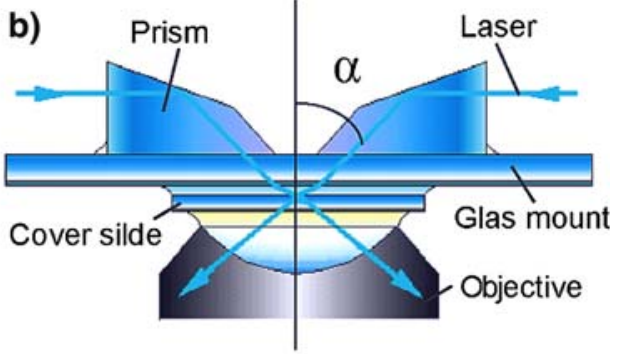

d)

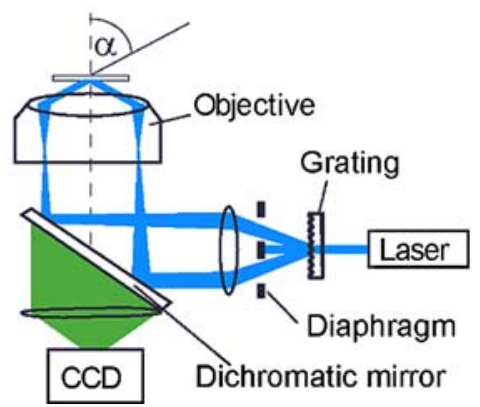


strongly demagnified. A practical set-up is shown in Fig. 4d. The diffracted beams are focused into the back focal plane of the objective to obtain plane waves in the specimen plane. The fluorescence signal collected by the objective is separated from the excitation light by a dichromatic mirror and a bandpass filter and recorded with a CCD camera.

To shift the harmonic excitation pattern one usually delays one laser beam of the interfering pair. To this end, piezo-actuated mirrors are inserted into the beam to adjust the optical path length (Frohn et al. 2000). Alternatively, one may insert an electrically tunable phase plate (Beck et al. 2008) that offers the additional advantage of maintaining a straight beam trajectory. When projecting a grating (Fig. 4d), translating the grating laterally shifts the illumination pattern, while rotating the grating (e.g., $45^{\circ}$ for a 2D-grating or $120^{\circ}$ and $240^{\circ}$ for a 1D-grating) adds spectral copies toward a more isotropic extended HELM passband compared to Fig. 3b (Gustafsson 2000).

\section{Applications and further developments of HELM}

\section{TIRF-HELM}

The benefits of combining TIRF microscopy with HELM (TIRF-HELM) have been early recognized by So and coworkers (Cragg and So 2000; Chung et al. 2006, 2007). In TIRF-HELM, the standing wave is formed by counterpropagating laser beams that experience total internal reflection inside the glass coverslip. Owing to the higher refractive index of the coverslip, the resulting evanescent standing wave emerging from the glass coverslip has a higher spatial frequency compared to standard HELM illumination in water or air. Hence, the lateral resolution can be improved by a factor of approx 2.5 , and even beyond using nanostructured substrates (Sentenac et al. 2008). The excitation intensity of TIRF-HELM is described by $I(x, y, z)=I_{0} e^{-d / z}\left[2+\cos \left(u_{x} x+\Delta \varphi_{x}\right)+\cos \left(u_{y} y+\Delta \varphi_{y}\right)\right]$, which is an evanescent wave in axial direction with a $2 \mathrm{D}$ cosine modulation laterally. The intensity distribution is plotted schematically in Fig. 5a and quantitatively for a glass/water interface at an illumination angle of $\alpha=63.2^{\circ}$ in Fig. $5 b$.

\section{Multi-color HELM}

Visualizing complex cellular mechanisms requires observation of several components tagged with different fluorescent markers simultaneously. A frequent aim of such studies is to judge whether certain components colocalize or act spatially separated from each other. Obviously, images with extended resolution as provided by the HELM technique show more detail and hence allow finer colocalization studies (Fig. 6). The HELM technique as described before for a single wavelength is easily extended to multi-color imaging. To this end, the laser light is conveniently launched by a single-mode optical fiber into the optics generating the harmonic excitation pattern (see Fig. 4). An acousto-optic modulator may be used to couple different wavelengths into the fiber and control their intensities. In multi-color HELM, the extent of the reconstructed passband will depend on the excitation and the emission wavelength. The emission wavelength determines the extent of the spectral components $\tilde{\Psi}_{i}$, i.e., the diameter of the individual disks depicted in Fig. 3b. The shift vector $\boldsymbol{u}$ is determined by the period of the standing wave pattern, i.e., by the excitation wavelength, the angle between the interfering laser beams, and the refractive index. Hence, even for single wavelength excitation, e.g., when using quantum dot labels, the resulting resolution will be higher for shorter emission wavelength than for longer emission wavelength. Using the grating approach for TIRF-HELM (Fig. 4d), excitation with different wavelengths requires adjustment of the diffraction angle, e.g., by a tunable grating (Beck et al. 2008) or spatial light modulator (Fiolka et al. 2008), to maintain the condition of total internal reflection. The period of the standing wave and its penetration depth into the sample will vary with wavelength. For example, the TIRF-HELM images shown in Fig. 6c, d were acquired with excitation wavelengths of 488 and $532 \mathrm{~nm}$. The periods $\Lambda$ of the standing wave pattern were
Fig. 5 Standing evanescent wave. a Set-up with two interfering laser beams undergoing total internal reflection in medium with refractive index $n_{1}$. b Calculated intensity distribution at a glass/ water interface for an illumination angle $\alpha=63.2^{\circ}$
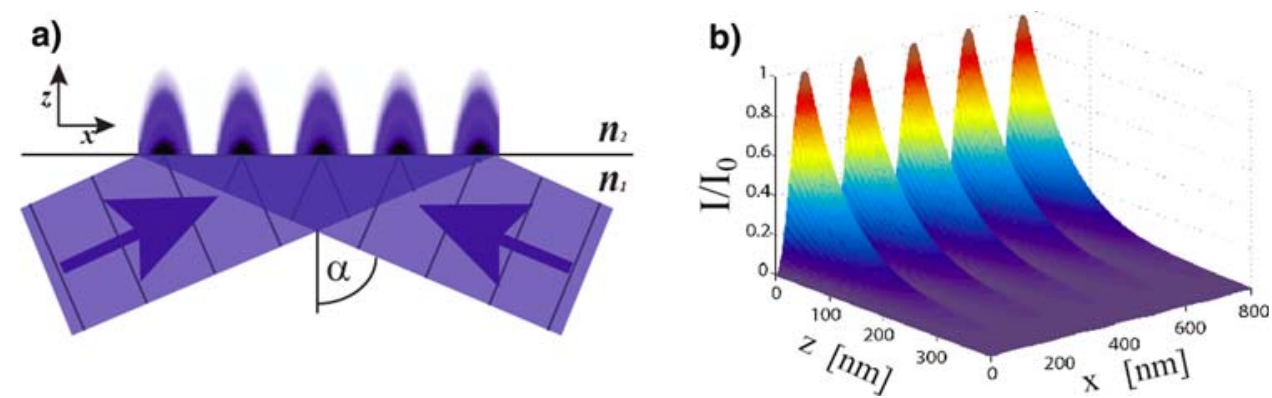

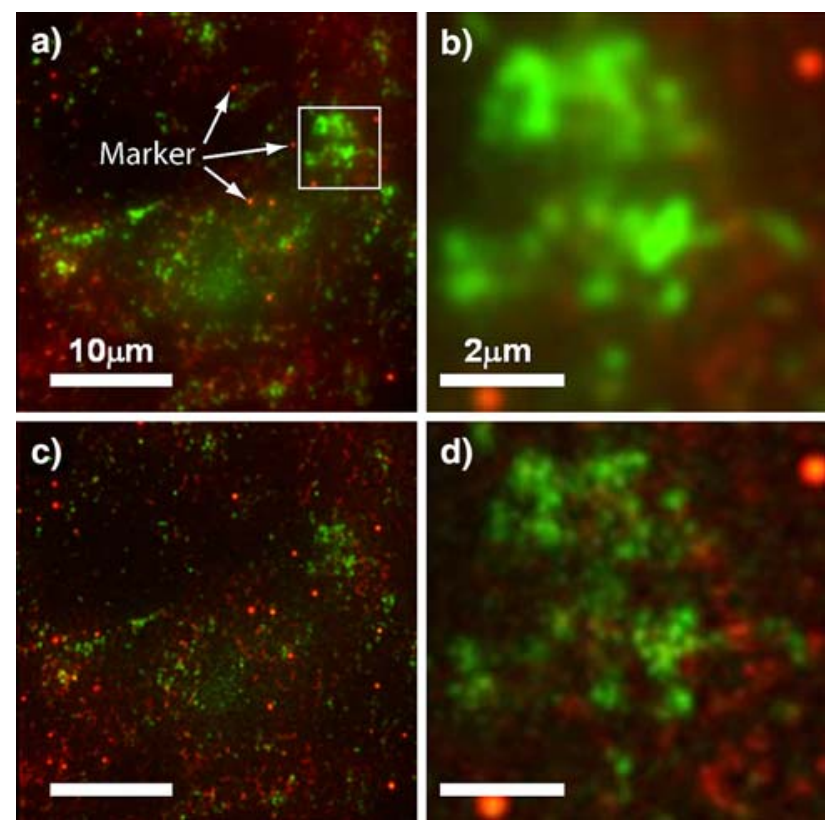

Fig. 6 Comparison of multi-color TIRF and TIRF-HELM. Normal African green monkey kidney fibroblast (CV-1) cells transfected with caveolin1-mGFP and dynamin2-mRFP. TetraSpeck markers attached to the coverslip were used to align the red and green channels (bright red spots, arrows). Conventional TIRF microscopy (a, b) and TIRF$\operatorname{HELM}(\mathbf{c}, \mathbf{d})$

$\Lambda_{488}=177 \mathrm{~nm}$ and $\Lambda_{532}=194 \mathrm{~nm}$, corresponding to penetration depths of $\mathrm{d}_{488}=104 \mathrm{~nm}$ and $\mathrm{d}_{532}=143 \mathrm{~nm}$, respectively.

Quantum dots (QDs) increasingly gain attention in biological imaging due to their long-term photostability compared to organic fluorophores. Blinking QDs (Nirmal et al. 1996; Jaiswal and Simon 2004), however, may interfere with the sequential acquisition of raw images in the HELM technique when the blinking frequency is in the range of the acquisition frame rate. Whereas blinking QDs may be detected in conventional microscopy as dimmer spots compared to non-blinking QDs, the blinking ones may be lost in the reconstruction of the extended resolution HELM image (Fig. 7).

\section{Saturated structured illumination microscopy (SSIM)}

Non-linear effects in fluorescence emission lead to higher harmonics in the image spectrum. Generally, non-linearities that are described by an $n$-th order polynomial result in the generation of $n$ harmonics. If the relation between excitation and emission is described by an exponential function, an infinite number of harmonics will occur. Such a relation would theoretically allow infinite resolution. Figure 8 illustrates the occurrence of higher harmonics in the fluorophore response when bleaching an initially homogenous fluorescent layer by a two-dimensional harmonic excitation field. Due to a non-linear bleaching rate, after a short time sharp intensity peaks of unbleached fluorophores form at the location of intensity zeros of the excitation field. Figure 8a-d shows the temporal evolution of the remaining active fluorophore population along with the corresponding image spectra (Fig. 8e).

Taking advantage of the non-linear response of fluorophores in the saturation regime (Heintzmann 2003; Gustafsson 2005), resolution in structured illumination
Fig. 7 HeLa cells labeled with actin-mGFP and quantum dots (QDs) binding to caveolin.

Conventional TIRF microscopy (a, a1) and TIRF-HELM (b, b1). Blinking QDs detected in the conventional image (a2) are lost in the HELM reconstruction (b2)
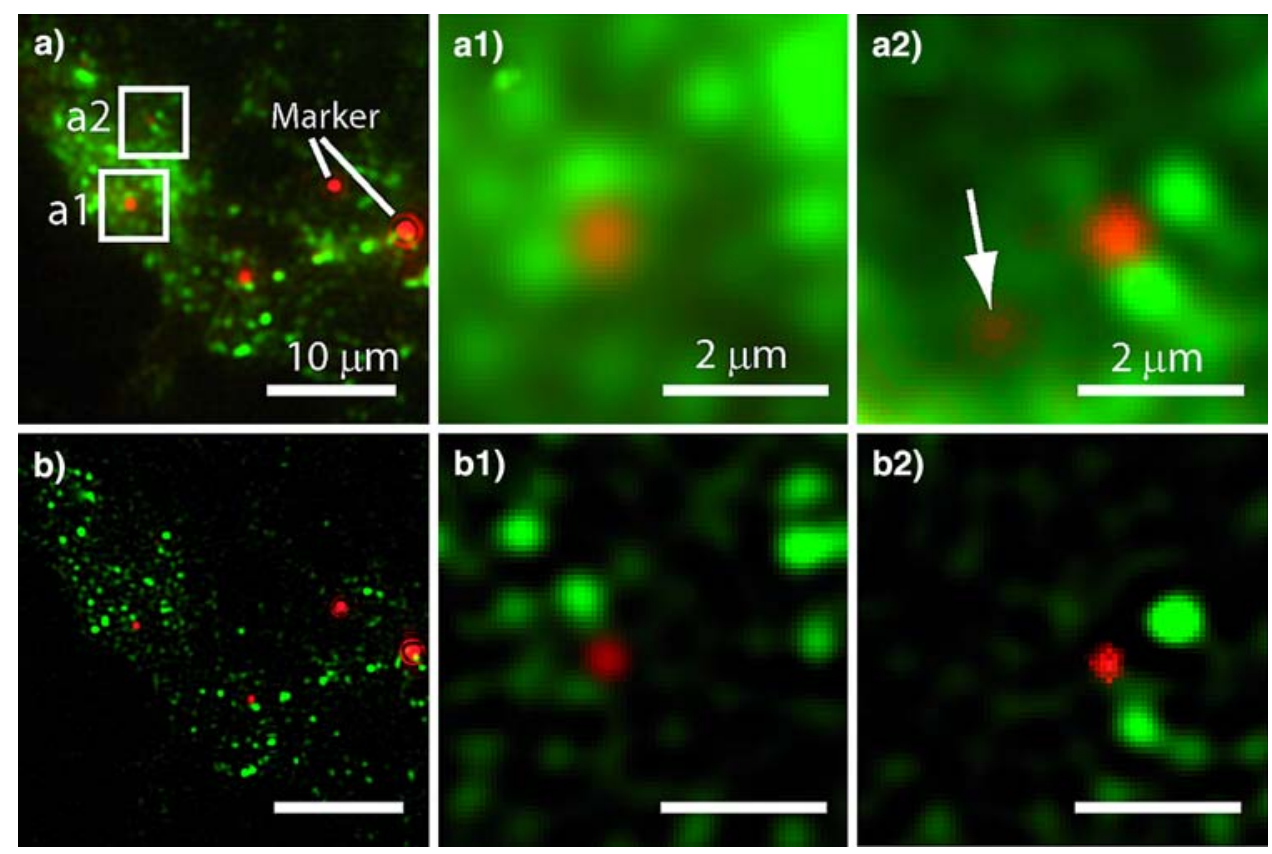
Fig. 8 Time series of structured field bleaching. Simulation of an initially homogenous fluorophore distribution exposed to a two-dimensional harmonic excitation field (a-d), and the corresponding spatial-frequency representation (e). The higher harmonics are enveloped by a Lorentzian
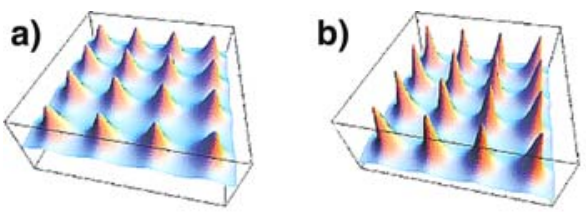

c)

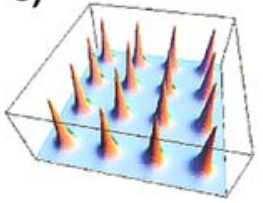

e)

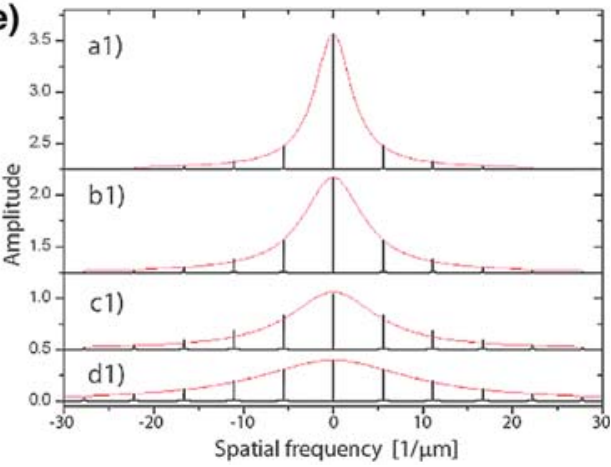

may be improved down to several $10 \mathrm{~nm}$. Saturated structured illumination microscopy (SSIM) applies very high excitation intensities to saturate the fluorophore response even in close vicinity of the zero intensities of the excitation pattern. As a result, most fluorophores are excited into a defined on-state and only small volumes, located at the intensity zeros, remain in the off-state. Similar to the HELM technique described above, a sequence of raw images with shifted excitation pattern is acquired to reconstruct the final extended resolution image. The required number of raw images increases with the order of harmonics to be resolved and the number of angular pattern orientations applied to achieve isotropic resolution. With enough laser intensity, this technique can theoretically achieve infinite resolution. In biological imaging, photostability and phototoxicity as well as long acquisition times will set a practical limit. With a picosecond laser an image resolution of $50 \mathrm{~nm}$ has been demonstrated (Fig. 9) (Gustafsson 2005).

Instead of excitation into saturation, photo-switchable fluorophores may be employed to create non-linearities in the fluorophore response (Enderlein 2005; Hofmann et al. 2005; Keller et al. 2007). Referred to as RESOLFT (reversible saturable/switchable optical fluorescence transition) a stunning resolution claim of $\lambda / 12$ has been reported in a field-scanning implementation (Schwentker et al. 2007). This real space field-scanning approach requires less post-processing compared to the reconstruction in Fourier space. Field-scanning, however, typically requires more images to correctly sample the specimen. We estimate at least twice the number of images to achieve the same resolution per direction.
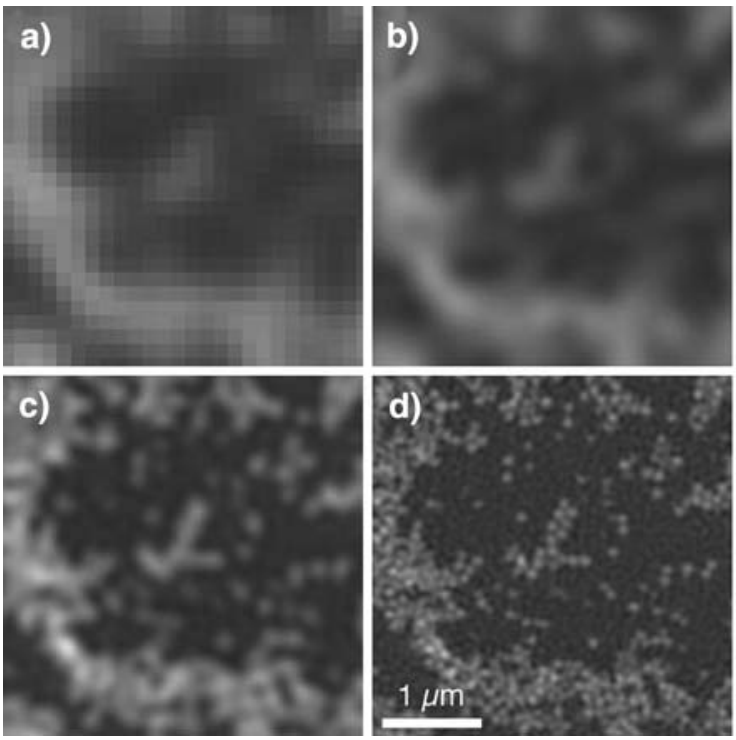

Fig. 9 A field of 50-nm fluorescent beads, imaged by conventional microscopy (a), conventional microscopy plus filtering (b), structured illumination with linear fluorophore response (c), and saturated structured illumination using illumination pulses of $5.3 \mathrm{~mJ} / \mathrm{cm}^{2}$ energy density (d). Taking into account three harmonic orders in

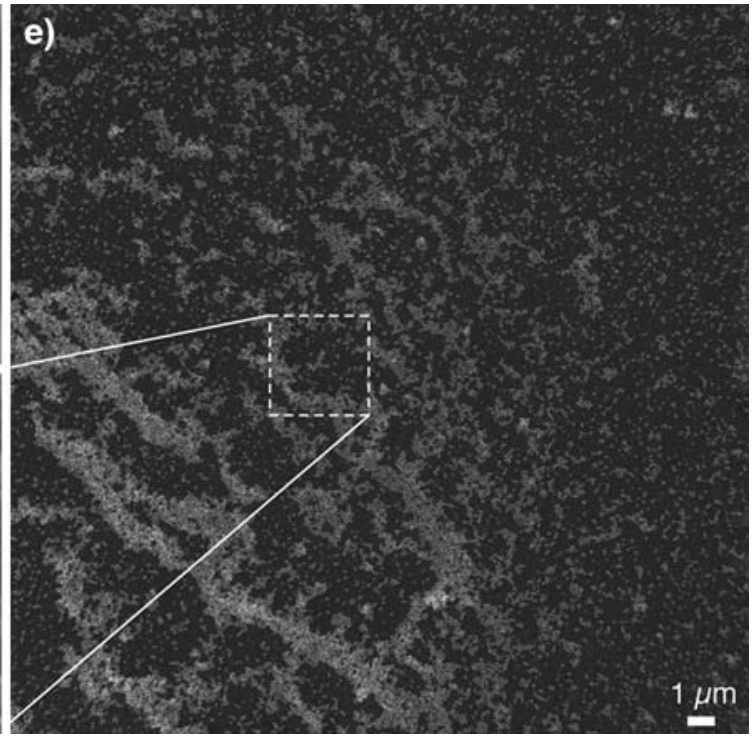

the processing, sequences of 9 images were recorded for each of the 12 equally spaced angular rotations of the line excitation pattern. Reprinted with permission from (Gustafsson 2005). Copyright (2005) Biophysical Society 

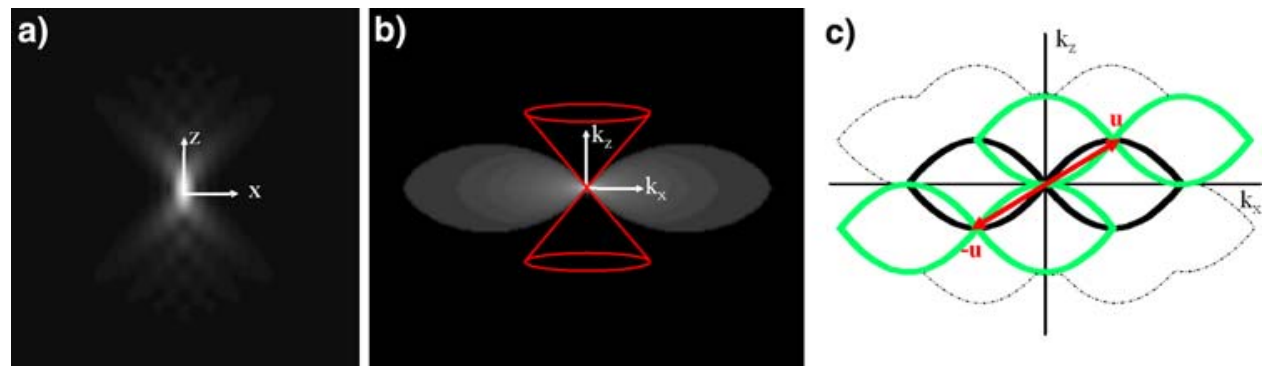

Fig. 10 a Cross-section of the 3D widefield PSF showing the central ellipsoid and the hourglass shaped structure responsible for out-offocus blur. The PSF is rotationally symmetric around the $z$ axis. b Cross-section of the 3D widefield OTF. Within a conical region around the $k_{\mathrm{z}}$ axis, no information is transferred. The OTF is rotationally symmetric around the $k_{\mathrm{z}}$ axis. $\mathbf{c}$ Resolution enhancement in 3D HELM: schematic representation of the original OTF (black envelope) and two copies (green envelope) obtained by structured illumination along the vector $\boldsymbol{u}$ (red arrow). The dash-dot envelope indicates the region that can be filled with five differently orientated interference patterns
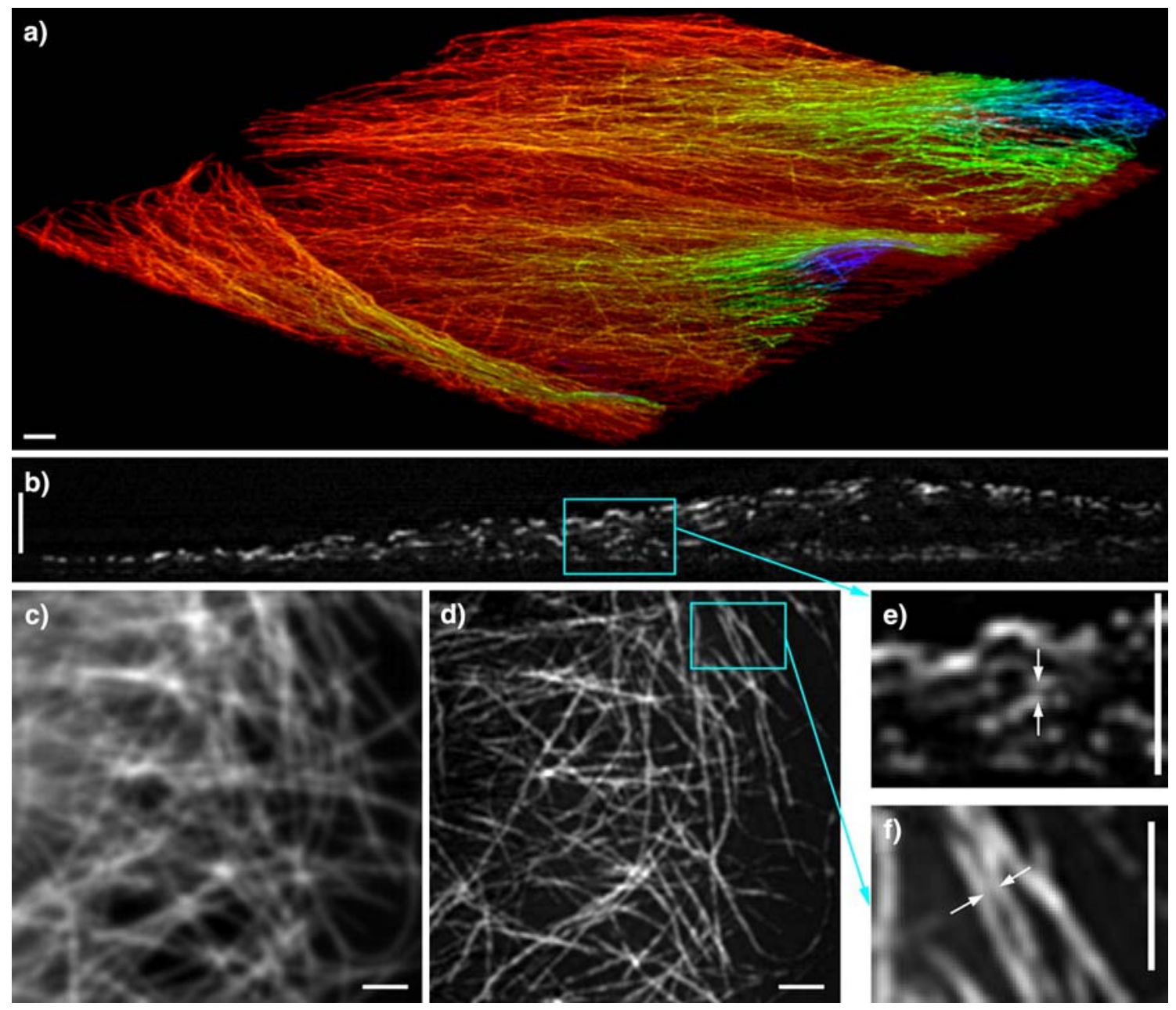

Fig. 11 The immunofluorescently labeled microtubule cytoskeleton in fixed HeLa cells imaged by I5S. a 3D rendering of a maximumintensity projection through the reconstructed data set, which captured portions of three neighboring cells. The hue indicates the axial position of each point, red-green-blue correspond to increasing distance from the cover glass. b A single axial $(y-z)$ section through the volume. The boxed region in $\mathbf{b}$ is magnified in $\mathbf{e}$, where the separation between the two parallel microtubules pointed to by the arrows is $106 \mathrm{~nm}$. c, d Comparison of lateral resolution between conventional microscopy (c) and I5S (d), using one lateral section from the volume. $\mathbf{f}$ Zoom of the boxed region in $\mathbf{d}$, where the separation between the two microtubules pointed to by arrows is $120 \mathrm{~nm}$. (All scale bars $1 \mu \mathrm{m}$.). Reprinted with permission from (Shao et al. 2008). Copyright (2008) National Academy of Sciences, USA 
Although the number of higher harmonics is infinite, at least in principle, only a finite number of harmonics will rise above the noise level. The difficulty in creating a real intensity zero also puts a practical limit on the achievable resolution with such non-linear techniques.

\section{D-HELM}

As mentioned in the "Introduction", axial resolution in widefield fluorescence microscopy is strongly limited. Figure 10a is an $x-z$ section of a 3D widefield PSF for fluorescent light emission. The elliptical shape along the $z$ coordinate causes the poor $z$-resolution. In addition to the central ellipsoid, the widefield PSF features an hourglass shaped structure. Owing to this structure, objects that are not in focus still contribute to the image, although extremely blurred. Thereby out-of-focus objects increase the background level and overshadow the in-focus image. The corresponding cross-section of the 3D-OTF, which is the Fourier transform of the 3D-PSF, is shown in Fig. 10b. Owing to the toroidal shape of the OTF, information in a conical region around the $k_{\mathrm{z}}$ axis, known as the missing cone, is not transferred. The missing cone is responsible for the poor axial resolution and the occurrence of out-of-focus blur in widefield microscopy. In confocal microscopy the missing cone is filled, improving axial resolution to about $700-800 \mathrm{~nm}$, which is still not sufficient to resolve typical biological structures along the $z$-direction.

Structured illumination with harmonic patterns that are tilted above the horizontal plane encodes three-dimensional information that is normally not transferred by the microscope. The tilted patterns create copies of the OTF that reach into the missing cone (Fig. 10c). Filling the missing cone improves axial resolution and deterministically removes out-of-focus blur. Frohn et al. (2001) suggested a set-up for 3D-HELM using two facing objectives and beam steering units for sequential illumination of the specimen with one-dimensional interference patterns.

Recently, Shao et al. (2008) presented first results of structured illumination in three dimensions. Their I5S microscope employs two opposing objectives to generate a complex three-dimensional interference pattern of multiple beams. The images of the two objectives are coherently superimposed as in the I5M microscope. The system achieves a near-isotropic resolution of about $100 \mathrm{~nm}$ in all directions, documented on complex biological samples. Figure 11 displays the three-dimensional microtubule cytoskeleton of HeLa cells imaged by I5S microscopy. The same group also presented a system using just one objective for illumination and detection (Gustafsson et al. 2008; Schermelleh et al. 2008), significantly reducing the complexity of the system while still reaching an axial resolution of approx $280 \mathrm{~nm}$.

\section{Conclusions}

Confocal microscopes becoming commercially available and a rapidly growing selection of fluorescent tags marked the beginning of a lasting renaissance of light microscopy in life science. The ability to visualize the three-dimensional dynamic architecture of cells with, at that time, unprecedented clarity paved the way to new insights into complex molecular mechanisms. Imaging three-dimensional specimens in widefield fluorescence microscopy somewhat remained a technique for experts, quite likely because in the past the computational methods necessary to reconstruct clear images appeared involved. Widefield microscopes, however, collect light emitted from the entire field of view in parallel and very efficiently since there are no pinholes cutting off light. We expect that the recent demonstrations of superb resolution and clarity achieved with structured illumination will now spur growing interest to apply widefield fluorescence microscopy in the study of ever finer structures and processes.

Acknowlegments We are grateful to Arnold Hayer and Dr. Helge Ewers from the Institute of Biochemistry at ETH Zurich for preparing labeled CV-1 and HeLa cells.

\section{References}

Abbe E (1873) Beiträge zur Theorie des Mikroskops und der mikroskopischen Wahrnehmung. Archiv für mikroskopische Anatomie 9:413-468

Bailey B, Farkas DL, Taylor DL, Lanni F (1993) Enhancement of axial resolution in fluorescence microscopy by standing-wave excitation. Nature 366:44-48

Beck M, Aschwanden M, Stemmer A (2008) Sub-100 nanometer resolution in total internal reflection fluorescence microscopy. J Microsc 232:99-105

Chung E, Kim D, So PTC (2006) Extended resolution wide-field optical imaging: objective-launched standing-wave total internal reflection fluorescence microscopy. Opt Lett 31:945-947

Chung E, Kim D, Cui Y, Kim Y-H, So PTC (2007) Two-dimensional standing wave total internal reflection fluorescence microscopy: superresolution imaging of single molecular and biological specimens. Biophys J 93:1747-1757

Cragg G, So PTC (2000) Lateral resolution enhancement with standing evanescent waves. Opt Lett 25:46-48

Enderlein J (2005) Breaking the diffraction limit with dynamic saturation optical microscopy. Appl Phys Lett 87:094105

Fiolka R, Beck M, Stemmer A (2008) Structured illumination in total internal reflection fluorescence microscopy using a spatial light modulator. Opt Lett 33:1629-1631

Frohn JT, Knapp HF, Stemmer A (2000) True optical resolution beyond the Rayleigh limit achieved by standing wave illumination. Proc Natl Acad Sci USA 97:7232-7236

Frohn JT, Knapp HF, Stemmer A (2001) Three-dimensional resolution enhancement in fluorescence microscopy by harmonic excitation. Opt Lett 26:828-830

Gustafsson MGL (2000) Surpassing the lateral resolution limit by afactor of two using structured illumination microscopy. J Microsc Oxf 198:82-87 
Gustafsson MGL (2005) Nonlinear structured-illumination microscopy: wide-field fluorescence imaging with theoretically unlimited resolution. Proc Natl Acad Sci USA 102:13081-13086

Gustafsson MG, Agard DA, Sedat JW (1995) Sevenfold improvement of axial resolution in 3D wide-field microscopy using two objective-lenses. Proc SPIE 2412:147-156

Gustafsson MG, Agard DA, Sedat JW (2000) Doubling the lateral resolution of wide-field fluorescence microscopy using structured illumination. Proc SPIE 3919:141-150

Gustafsson MGL, Shao L, Carlton PM, Wang CJR, Golubovskaya IN, Cande WZ, Agard DA, Sedat JW (2008) Three-dimensional resolution doubling in widefield fluorescence microscopy by structured illumination. Biophys J 94:4957-4970

Heintzmann R (2003) Saturated patterned excitation microscopy with two-dimensional excitation patterns. Micron 34:283-291

Heintzmann R, Cremer CG (1999) Laterally modulated excitation microscopy: improvement of resolution by using a diffraction grating. In: Bigio IJ, Schneckenburger H, Slavik J, Svanberg K, Viallet PM (eds) Optical Biopsies and Microscopic Techniques III, 1999/01/19/. SPIE, Stockholm, pp 185-196

Heintzmann R, Jovin TM, Cremer C (2002) Saturated patterned excitation microscopy-a concept for optical resolution improvement. J Opt Soc Am A Opt Image Sci Vis 19:1599-1609

Hell SW, Stelzer EHK (1992) Fundamental improvement of resolution with a 4Pi-confocal fluorescence microscope using twophoton excitation. Opt Commun 93:277-282

Hell SW, Wichmann J (1994) Breaking the diffraction resolution limit by stimulated emission: stimulated emission depletion microscopy. Opt Lett 19:780-782

Hofmann M, Eggeling C, Jakobs S, Hell SW (2005) Breaking the diffraction barrier in fluorescence microscopy at low light intensities by using reversibly photoswitchable proteins. Proc Natl Acad Sci USA 102:17565-17569

Jaiswal JK, Simon SM (2004) Potentials and pitfalls of fluorescent quantum dots for biological imaging. Trends Cell Biol 14:497-504

Keller J, Schönle A, Hell SW (2007) Efficient fluorescence inhibition patterns for RESOLFT microscopy. Opt Express 15:3361-3371

Klar TA, Jakobs S, Dyba M, Egner A, Hell SW (2000) Fluorescence microscopy with diffraction resolution barrier broken by stimulated emission. Proc Natl Acad Sci USA 97:8206-8210
Lukosz W (1966) Optical systems with resolving powers exceeding classical limit. J Opt Soc Am 56:1463-1472

Minsky M (1988) Memoir on inventing the confocal scanning microscope. Scanning 10:128-138

Nirmal M, Dabbousi BO, Bawendi MG, Macklin JJ, Trautman JK, Harris TD, Brus LE (1996) Fluorescence intermittency in single cadmium selenide nanocrystals. Nature 383:802-804

Schaefer LH, Schuster D, Schaffer J (2004) Structured illumination microscopy: artefact analysis and reduction utilizing a parameter optimization approach. J Microsc 216:165-174

Schermelleh L, Carlton PM, Haase S, Shao L, Winoto L, Kner P, Burke B, Cardoso MC, Agard DA, Gustafsson MGL, Leonhardt H, Sedat JW (2008) Subdiffraction multicolor imaging of the nuclear periphery with 3D structured illumination microscopy. Science 320:1332-1336

Schwentker MA, Bock H, Hofmann M, Jakobs S, Bewersdorf J, Eggeling C, Hell SW (2007) Widefield subdiffraction RESOLFT microscopy using fluorescent protein photoswitching. Microsc Res Tech 70:269-280

Sentenac A, Belkebir K, Giovannini H, Chaumet PC (2008) Subdiffraction resolution in total internal reflection fluorescence microscopy with a grating substrate. Opt Lett 33:255-257

Shao L, Isaac B, Uzawa S, Agard DA, Sedat JW, Gustafsson MGL (2008) I5S: Widefield light microscopy with 100-nm-scale resolution in three dimensions. Biophys J 94:4971-4983

So PTC, Kwon HS, Dong CY (2001) Resolution enhancement in standing-wave total internal reflection microscopy: a pointspread-function engineering approach. J Opt Soc Am A Opt Image Sci Vis 18:2833-2845

Stephens DJ, Allan VJ (2003) Light microscopy techniques for live cell imaging. Science 300:82-86

Taylor DL, Wang Y-L (1980) Fluorescently labelled molecules as probes of the structure and function of living cells. Nature 284:405-410

White J, Amos W, Fordham M (1987) An evaluation of confocal versus conventional imaging of biological structures by fluorescence light microscopy. J Cell Biol 105:41-48 